\title{
Does Irrigation with Treated and Untreated Wastewater Increase Antimicrobial Resistance in Soil and Water: A Systematic Review
}

\author{
Stacy Slobodiuk ${ }^{1, *}$, Caitlin Niven ${ }^{1}$, Greer Arthur ${ }^{2}{ }^{\oplus}$, Siddhartha Thakur ${ }^{2}$ and Ayse Ercumen ${ }^{1}$ \\ 1 Department of Forestry and Environmental Resources, North Carolina State University, \\ Raleigh, NC 27695, USA; cgniven@ncsu.edu (C.N.); aercume@ncsu.edu (A.E.) \\ 2 Department of Population Health and Pathobiology, North Carolina State University, \\ Raleigh, NC 27695, USA; gkarthur@ncsu.edu (G.A.); sthakur@ncsu.edu (S.T.) \\ * Correspondence: sslobod@ncsu.edu; Tel.: +1-704-453-1219
}

check for updates

Citation: Slobodiuk, S.; Niven, C.; Arthur, G.; Thakur, S.; Ercumen, A. Does Irrigation with Treated and Untreated Wastewater Increase Antimicrobial Resistance in Soil and Water: A Systematic Review. Int. J. Environ. Res. Public Health 2021, 18, 11046. https://doi.org/10.3390/ ijerph182111046

Academic Editor: Farooq Sher

Received: 30 August 2021

Accepted: 12 October 2021

Published: 21 October 2021

Publisher's Note: MDPI stays neutral with regard to jurisdictional claims in published maps and institutional affiliations.

Copyright: (c) 2021 by the authors. Licensee MDPI, Basel, Switzerland. This article is an open access article distributed under the terms and conditions of the Creative Commons Attribution (CC BY) license (https:// creativecommons.org/licenses/by/ $4.0 /)$.

\begin{abstract}
Population growth and water scarcity necessitate alternative agriculture practices, such as reusing wastewater for irrigation. Domestic wastewater has been used for irrigation for centuries in many historically low-income and arid countries and is becoming more widely used by high-income countries to augment water resources in an increasingly dry climate. Wastewater treatment processes are not fully effective in removing all contaminants, such as antimicrobial resistant bacteria (ARB) and antimicrobial resistance genes (ARGs). Literature reviews on the impact of wastewater irrigation on antimicrobial resistance (AMR) in the environment have been inconclusive and mostly focused on treated wastewater. We conducted the first systematic review to assess the impact of irrigation with both treated or untreated domestic wastewater on ARB and ARGs in soil and adjacent water bodies. We screened titles/abstracts of 3002 articles, out of which 41 were screened in full text and 26 were included in this review. Of these, thirteen investigated irrigation with untreated wastewater, and nine found a positive association with ARB/ARGs in soil. Out of thirteen studies focused on treated wastewater, six found a positive association with ARB/ARGs while six found mixed/negative associations. Our findings demonstrate that irrigation with untreated wastewater increases AMR in soil and call for precautionary action by field workers, their families, and consumers when untreated wastewater is used to irrigate crops. The effect of irrigation with treated wastewater was more variable among the studies included in our review, highlighting the need to better understand to what extent AMR is disseminated through this practice. Future research should assess factors that modify the effect of wastewater irrigation on AMR in soil, such as the degree and type of wastewater treatment, and the duration and intensity of irrigation, to inform guidelines on the reuse of wastewater for irrigation.
\end{abstract}

Keywords: antimicrobial resistance; resistance genes; resistant bacteria; persistent organic pollutants; wastewater irrigation; wastewater reuse; water scarcity; water insecurity; untreated municipal wastewater; treated municipal wastewater

\section{Introduction}

Consequences of an ever-growing global population, such as water pollution, climate change, and unevenly distributed water resources, have led to limitations in accessing clean freshwater, driving the need for the reuse and recycling of water resources. Agriculture is the largest user of freshwater and accounts for almost $75 \%$ of water use [1]. With the world's population estimated to reach 10 billion within the next 30 years, agricultural production is predicted to increase by $70 \%$, putting further strain on freshwater resources [2]. Almost $50 \%$ of the world's population uses polluted water sources for agricultural irrigation, and 20 million hectares are estimated to be irrigated with wastewaters [3]. Wastewater has been used in agriculture for centuries in many cities around the world that have a 
historically low accumulation of rainwater. It is also an increasingly critical alternative source of water in countries that are most impacted by water scarcity, especially those which rely on agriculture for income. For many low-income countries, reusing untreated wastewater is one of the few affordable alternatives to the advanced processes that occur in most wastewater treatment plants in high-income countries [4]; however, increasing stress on water resources has also led high-income countries to reuse domestic wastewater. For example, the U.S. reuses $4 \%$ of its treated wastewater, and some states rely on treated wastewater extensively, such as California and Florida, which use approximately half of their treated wastewater for agriculture [5]. China uses reclaimed wastewater for multiple applications, with one-third of its reclaimed wastewater going towards agricultural irrigation [6]. Irrigating crops with wastewater can also be beneficial as it supplies nutrients to the soil, reducing the need for farmers to purchase fertilizer [6]. In addition to agriculture, irrigation with treated wastewater is also used for landscaping and urban parks.

Wastewater can also contain high concentrations of heavy metals, pathogens, pharmaceuticals, plastic additives, and other contaminants. Contaminants can adversely affect plant growth when wastewater is applied to crops [7]. Human exposure to wastewater contaminants can also be harmful, and agricultural reuse of wastewater has been associated with health risks. Exposure to wastewater through agricultural irrigation has been linked to enteric diseases such as salmonellosis, shigellosis, cholera, giardiasis, amoebiasis, hepatitis A infections, and viral enteritis among farmers, their families, those living close to wastewater irrigation areas, and consumers of crops irrigated with wastewater [8]. Farmers working in fields that use untreated wastewater for irrigation have also reported experiencing skin irritation, rashes, and dermatitis [8]. Adequate treatment of wastewater prior to agricultural application can alleviate some of these health concerns. However, wastewater treatment processes are not fully effective in removing all contaminants. Contaminants of particular concern include pharmaceuticals, personal care products and antibiotic residues, as well as antimicrobial resistant bacteria (ARB) and antimicrobial resistance genes (ARGs) [9]. Antibiotics are detected in treated wastewater effluent [10] and ARB/ARGs can withstand or even proliferate at treatment plants [11]. Wastewater irrigation can lead to continuous exposure of the irrigated fields to a variety of antibiotics, which can prompt the emergence of resistant strains (Figure 1). ARB in wastewater deposited onto soils by irrigation can also elicit the transfer of ARGs between wastewater bacteria and native soil communities [12]. Crops planted in soil irrigated with wastewater can take up ARB/ARGs [13] and pose a risk of spreading AMR to consumers [14,15]. There is also the potential of ARB/ARG contamination in water bodies that are adjacent to wastewater-irrigated soils [16].

Antibiotics are considered persistent organic pollutants of emerging concern due to their known lasting effects on aquatic environments [17]. Effects of antibiotics on other environmental media are not as well known. Previous non-systematic reviews have provided mixed results on how wastewater irrigation affects AMR in soil. One review concluded that soils irrigated with treated wastewater do not demonstrate an increase in ARB and ARGs [11]. Two other reviews had inconclusive results [12,18]. These reviews were mostly focused on irrigation with treated wastewater and included few studies on irrigation with untreated wastewater. We conducted a systematic literature review to assess the effect of irrigation with both treated and untreated wastewater on the prevalence and abundance of ARB and ARGs in soils and adjacent water bodies. While wastewater from animal sources is often also used for irrigation, and both manure and municipal fecal sludge are used for soil amendment, we focused our review on irrigation with municipal domestic wastewater, either alone or combined with other waste streams. 


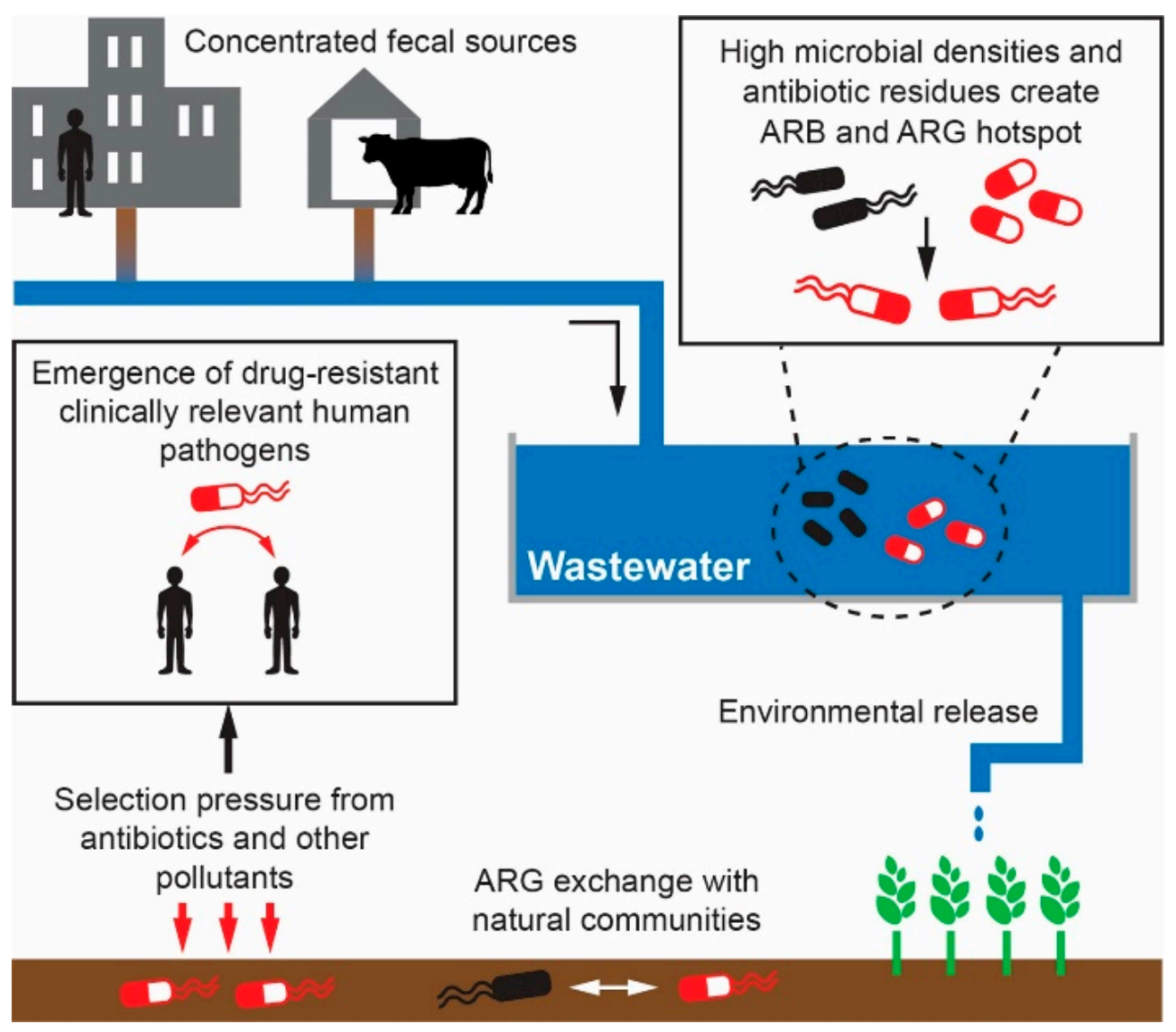

Figure 1. Role of wastewater irrigation in the emergence and spread of antimicrobial resistant bacteria (ARB) and antimicrobial resistance genes (ARGs) in the environment.

\section{Materials and Methods}

\subsection{Search Strategy}

We searched the PubMed, Web of Science, CAB Direct, and Agricultural and Environmental Science databases and conducted a search for gray literature in Science.gov. We developed search terms to denote treated and untreated wastewater (e.g., wastewater, sewage, effluent, reclaimed wastewater), agricultural processes that use wastewater (irrigation, agriculture), outcomes of interest (antimicrobial/antibiotic resistance), and environmental reservoirs of interest (e.g., soil, field, surface water, groundwater; Table S1). More detailed information on the PubMed search string can be found in the supplementary information (Text S1). The search was conducted in November 2020. References identified in the database search were imported into Covidence software, where duplicates were removed. Titles and abstracts of the articles were screened using our inclusion and exclusion criteria. For any review articles identified during the title/abstract screening, we screened the bibliographies to identify additional relevant studies. Articles short-listed during the title/abstract screening were reviewed in full text to determine eligibility.

\subsection{Inclusion and Exclusion Criteria}

We included studies if they detected or quantified ARB or ARGs in soils irrigated with wastewater or in water bodies adjacent to wastewater-irrigated soils. Studies were included if any form of treated or untreated domestic wastewater (alone, mixed with industrial/other waste streams, or diluted in an ambient water body after discharge) was used for irrigation. We excluded (1) studies that focused on application or amendment with sewage sludge or biosolids and with solely non-human waste (agriculture waste, dairy waste, piggery waste, manure), (2) studies that covered AMR in aquaculture and marine 
environments, and (3) experimental lab studies or studies conducted with irrigation water artificially spiked with antibiotics and/or bacteria that would be found in wastewater.

\subsection{Data Extraction and Synthesis}

We extracted relevant data from eligible full-text articles using Microsoft Excel. Extraction was conducted by two reviewers (SS, CN) independently to ensure accuracy, and any discrepancies were resolved by discussion. We reported data on study location, study design, type and treatment of wastewater, duration of irrigation, type of samples tested, analytical methods, prevalence and abundance of ARB and ARGs detected, and additional relevant environmental factors (e.g., $\mathrm{pH}$, soil moisture, seasonality). We qualitatively synthesized data from all eligible articles to separately assess the effect of irrigation with treated and untreated municipal wastewater on the prevalence and abundance of specific ARB/ARGs in soil and adjacent water bodies. We also investigated the effect of additional environmental variables on AMR in soil and water. Our PRISMA checklist can be found in the supplementary information (Table S2).

\section{Results}

We screened the titles/abstracts of 3002 studies and reviewed the full texts of 41 studies. Based on our inclusion/exclusion criteria, a total of 26 studies were eligible to be included in the review (Figure 2). The eligible studies were conducted between 2003 to 2020. Eight studies were conducted in high-income countries (Israel, Spain, Australia, Germany, US) while eighteen studies were based in low-and middle-income countries (Nigeria, Cameroon, Burkina Faso, Egypt, India, Mexico, China). Most studies focused on agricultural fields while four studies investigated urban parks and one study focused on a recharge basin. Twenty-five studies focused on soil samples and only one study investigated subsoil pore water samples.

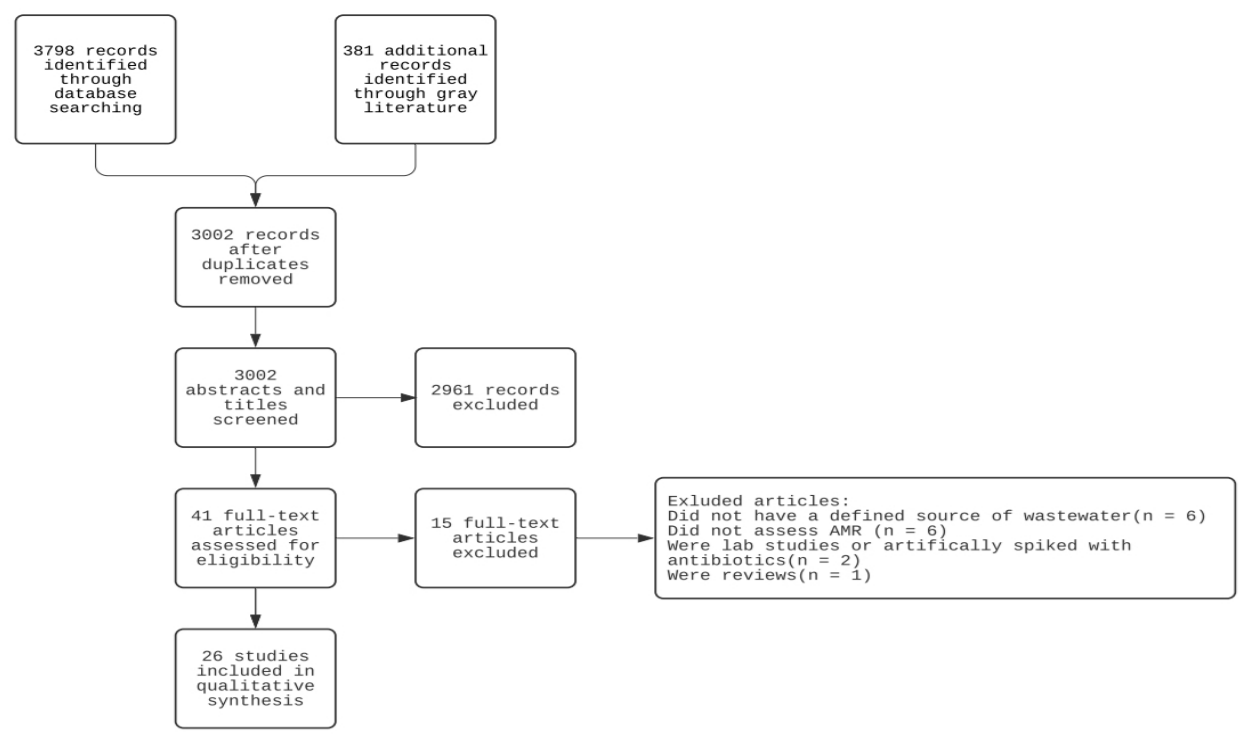

Figure 2. Flowchart of literature review and screening.

Thirteen studies focused on irrigation with untreated municipal wastewater and another thirteen studies focused on treated municipal wastewater. The studies included irrigation both directly with wastewater and indirectly using water from an ambient water body that receives wastewater or treated wastewater effluent. Soils irrigated with freshwater, rainfed fields, and pristine soils from remote areas (e.g., national parks) were used as a comparison group. The duration of wastewater irrigation ranged from 1.5 years [18] to 100 years [19-21]. Studies used a mix of culture-based and molecular methods and reported the prevalence of antimicrobial-resistant isolates and both absolute abundances 
of ARGs and relative abundances normalized by $16 \mathrm{~S}$ rRNA gene counts. The most commonly detected ARB included fecal bacteria and native soil bacteria, such as Escherichia coli, Enterococcus, Azotobacter chroococcum, Pseudomonas spp., and Flavobacteria. Studies assessed resistance to a wide range of antibiotics, including tetracycline, ampicillin, and ciprofloxacin. Commonly investigated ARGs included tetracycline resistance genes, sulfonamide resistance genes, quinolone resistance genes, and beta-lactamase genes. Some studies focused on detecting genetic elements involved in horizontal gene transfer, such as class 1 integrons (intl1).

\subsection{Irrigation with Untreated Wastewater}

Out of thirteen studies focused on untreated wastewater, one studied solely domestic wastewater, seven studied wastewater that was a combination of domestic and industrial, hospital, agriculture, market or slaughterhouse waste, and five referred to municipal wastewater without specifying the content (Table 1). Three studies had no comparison group to allow assessment of associations but detected ARB in soils irrigated with untreated wastewater [22-24]. Of the ten studies with a comparison group, nine found that wastewater irrigation was associated with increased ARB/ARGs in soil (Table 1). In one of these nine studies, the wastewater came indirectly from a waterbody [25]. Additional details of the studies are provided in the Supplemental Information (Table S3).

Four studies were conducted in Mezquital Valley in Mexico, one of the world's largest wastewater irrigation systems, where untreated wastewater from Mexico City has been used to irrigate farmlands for 100 years [26]. All of these studies found a positive association between wastewater irrigation and ARB/ARGs in soil. Studies at this site also took advantage of the long history of wastewater irrigation to assess whether ARB/ARGs in soil increase with increasing duration of irrigation. One study found substantially more isolates resistant to at least one antibiotic in wastewater-irrigated fields $(51 \%)$ than in rainfed fields $(6 \%)$ and a higher prevalence $(25 \%)$ of isolates resistant to $\geq 2$ antibiotics in wastewater-irrigated fields than in rainfed fields (6\%) [20]. Another study found the absolute abundance of sul1 genes to be 150-1500 times higher and sul2 genes 50-520 times higher in wastewater-irrigated soils than in rainfed soils; the relative abundance of both genes was also higher in wastewater-irrigated soils. While the absolute abundance of both genes increased with increasing years of irrigation, the relative abundance did not; soils irrigated for 100 years did not contain more sul1 and sul2 genes on the relative scale compared to soils irrigated with wastewater for 1.5 years [19]. A similar study at this site showed significant positive correlations between absolute gene abundance and years of irrigation for intl1, kor $B$, tet $W$, aad $A$, and qacE + qacE $\Delta 1$ (quaternary ammonium compound resistance) genes while the relative abundance of these genes did not vary with duration of wastewater irrigation [21]. A fourth study from Mezquital Valley compared a field that has been irrigated with untreated wastewater for over 80 years to a rainfed field that had never been irrigated. Soil samples from the wastewater-irrigated field had an absolute abundance of $3.3 \times 10^{6}$ gene copies of sul1 genes per $\mathrm{g}$ of soil compared to $3.1 \times 10^{5}$ gene copies per $\mathrm{g}$ in samples from the rainfed field while sul2 genes were only detected in the wastewater-irrigated field [27]. In a further experiment in the same study, where soil cores from both fields were irrigated with wastewater with and without sulfamethoxazole and ciprofloxacin, the relative abundance of sul1 genes in soil from the rainfed field increased by up to 3 orders of magnitude after the irrigation experiment, while it increased by $<1$ order of magnitude in soil from the wastewater-irrigated field. 
Table 1. Characteristics of studies on irrigation with untreated wastewater.

\begin{tabular}{|c|c|c|c|c|c|c|c|}
\hline Author and Year & Location & WWI Site & WWI Duration & Comparison Site & Organism & AMR Mechanism Investigated & Reference \\
\hline Aleem et al., 2003 & India & $\begin{array}{l}\text { Field irrigated with } \\
\text { untreated industrial } \\
\text { wastewater mixed with } \\
\text { domestic sewage }\end{array}$ & 10 years & $\begin{array}{l}\text { Field irrigated with } \\
\text { groundwater }\end{array}$ & $\begin{array}{l}\text { Azotobacter } \\
\text { chroococcum isolates }\end{array}$ & $\begin{array}{l}\text { Resistance against: Amoxycillin, } \\
\text { Cloxacillin, Co-trimoxazole, } \\
\text { Doxycycline, Methicillin, } \\
\text { Nitrofurantoin, Polymyxin-B, } \\
\text { Rifampicin, Streptomycin, } \\
\text { Sulphadiazine, Tetracycline }\end{array}$ & [22] \\
\hline Ansari et al., 2007 & India & $\begin{array}{l}\text { Field irrigated with } \\
\text { untreated industrial } \\
\text { wastewater mixed with } \\
\text { domestic sewage }\end{array}$ & $>20$ years & None & Bacterial isolates & $\begin{array}{l}\text { Resistance against: Ampicillin, } \\
\text { Chloramphenicol, Ciprofloxacin, } \\
\text { Co-trimoxazole, Doxycycline, } \\
\text { Gentamicin, Kanamycin, Nalidixic acid, } \\
\text { Neomycin, Streptomycin, Tetracycline }\end{array}$ & [23] \\
\hline Bahig et al., 2008 & Egypt & $\begin{array}{l}\text { Field irrigated with } \\
\text { untreated wastewater }\end{array}$ & Not reported & $\begin{array}{l}\text { Field irrigated with } \\
\text { canal water }\end{array}$ & Bacterial isolates & $\begin{array}{l}\text { Resistance against: Ampicillin, } \\
\text { Tetracycline, Kanamycin }\end{array}$ & [28] \\
\hline Bougnom et al., 2019 & $\begin{array}{l}\text { Burkina Faso, } \\
\text { Cameroon }\end{array}$ & $\begin{array}{l}\text { Field irrigated with } \\
\text { untreated domestic } \\
\text { wastewater mixed with } \\
\text { hospital, agriculture, } \\
\text { market and } \\
\text { slaughterhouse waste }\end{array}$ & 20 years & Non-irrigated field & $\mathrm{N} / \mathrm{A}^{\mathrm{a}}$ & $\begin{array}{l}\text { ARGs encoding: Antibiotic inactivation } \\
\text { enzymes, antibiotic target replacement, } \\
\text { antibiotic target protection, efflux } \\
\text { pumps }\end{array}$ & [3] \\
\hline Bougnom et al., 2020 & $\begin{array}{l}\text { Same as } \\
\text { above }\end{array}$ & Same as above & Same as above & Same as above & Same as above & $\begin{array}{l}\text { Non-targeted ARGs and } \\
\text { Enterobacteriaceae plasmid replicons }\end{array}$ & [30] \\
\hline Broszat et al., 2014 & Mexico & $\begin{array}{l}\text { Field irrigated with } \\
\text { untreated municipal } \\
\text { wastewater }\end{array}$ & $\begin{array}{l}8,10,85, \text { and } \\
100 \text { years }\end{array}$ & Rain-fed field & Bacterial isolates & $\begin{array}{l}\text { Resistance against: Ampicillin, } \\
\text { Chloramphenicol, Erythromycin, } \\
\text { Gentamicin, Kanamycin, Oxacillin, } \\
\text { Streptomycin, Ciprofloxacin, } \\
\text { Doxycycline, Tetracycline, Vancomycin, } \\
\text { Sulfamethoxazole } \\
\text { ARGs: Sulfonamide }(s u l) \text { and } \\
\text { fluoroquinolone }(q n r) \text { resistance genes }\end{array}$ & [20] \\
\hline Dalkmann et al., 2012 & Mexico & $\begin{array}{l}\text { Field irrigated with } \\
\text { untreated municipal } \\
\text { wastewater }\end{array}$ & $\begin{array}{l}1.5,3,6,8,85, \\
\text { and } 100 \text { years }\end{array}$ & Rain-fed field & $\mathrm{N} / \mathrm{A}^{\mathrm{a}}$ & 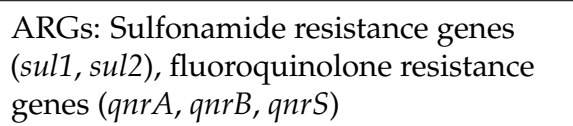 & [19] \\
\hline
\end{tabular}


Table 1. Cont

\begin{tabular}{|c|c|c|c|c|c|c|c|}
\hline Author and Year & Location & WWI Site & WWI Duration & Comparison Site & Organism & AMR Mechanism Investigated & Reference \\
\hline Jechalke et al., 2015 & Mexico & $\begin{array}{l}\text { Field irrigated with } \\
\text { untreated municipal } \\
\text { wastewater }(65 \% \text { domestic } \\
\text { sewage, } 20 \% \text { service sector } \\
\text { waste, } 15 \% \text { industrial } \\
\text { waste) }\end{array}$ & $\begin{array}{l}1.5,3,6,8,85 \\
\text { and } 100 \text { years }\end{array}$ & Rain-fed field & $\mathrm{N} / \mathrm{A}^{\mathrm{a}}$ & $\begin{array}{l}\text { ARGs: tet } W \text {, tet } Q, \text { aad } A, q a c E+\text { qacE } \Delta 1 \\
\text { Mobile genetic elements: intI1, } \\
\text { IncP-1plasmids (korB) }\end{array}$ & [21] \\
\hline Lüneberg et al., 2017 & Mexico & $\begin{array}{l}\text { Field irrigated with } \\
\text { untreated wastewater }\end{array}$ & $>80$ years & Rain-fed field & $\mathrm{N} / \mathrm{A}^{\mathrm{a}}$ & ARGs: sul1, sul2, qnrB, qnrS & [27] \\
\hline Malik and Aleem 2011 & India & $\begin{array}{l}\text { Field irrigated with } \\
\text { untreated industrial } \\
\text { wastewater mixed with } \\
\text { domestic sewage }\end{array}$ & 10 years & $\begin{array}{l}\text { Field irrigated with } \\
\text { groundwater }\end{array}$ & $\begin{array}{l}\text { Pseudomonas spp. } \\
\text { isolates }\end{array}$ & $\begin{array}{l}\text { Resistance against: Amoxycillin, } \\
\text { Ampicillin, Chloramphenicol, } \\
\text { Ciprofloxacin, Cloxacillin, } \\
\text { Cotrimoxazole, Doxycycline, } \\
\text { Erythromycin, Gentamicin, Kanamycin, } \\
\text { Methicillin, Nalidixic acid, Neomycin, } \\
\text { Nitrofurantoin, Polymyxin-B, } \\
\text { Rifampicin, Streptomycin, } \\
\text { Sulphadiazine, Tetracycline }\end{array}$ & [31] \\
\hline Pan and Chu 2018 & China & $\begin{array}{l}\text { (1) Fields irrigated with } \\
\text { untreated domestic } \\
\text { wastewater } \\
\text { (2) Fields irrigated with } \\
\text { fishpond water }\end{array}$ & $>20$ years & $\begin{array}{l}\text { Field with no } \\
\text { cultivation }\end{array}$ & $\mathrm{N} / \mathrm{A}^{\mathrm{a}}$ & $\begin{array}{l}\text { ARGs: Tetracycline }(\text { tet } A, \text { tet } B, \text { tet } C \text {, tet } E \text {, } \\
\text { tet } M, \text { tet } O \text {, tet } S, \text { tet } X) \text { and sulfonamide } \\
\text { resistance genes }(s u l 1, \text { sul } 2, \text { sul } 3)\end{array}$ & [29] \\
\hline Shafiani and Malik 2013 & India & $\begin{array}{l}\text { Field irrigated with } \\
\text { untreated industrial } \\
\text { wastewater mixed with } \\
\text { domestic sewage }\end{array}$ & 10 years & None & $\begin{array}{l}\text { Pseudomonas spp. } \\
\text { isolates }\end{array}$ & $\begin{array}{l}\text { Resistance against: Amoxycillin, } \\
\text { Chloramphenicol, Cloxacillin, } \\
\text { Doxycycline, Methicillin, Nalidixic acid, } \\
\text { Tetracycline }\end{array}$ & [24] \\
\hline
\end{tabular}

WWI: Wastewater irrigation; AMR: Antimicrobial resistance; ARG: Antimicrobial resistance gene. ${ }^{\text {a }}$ No specific target organism, DNA extracted directly from soil. 
Other investigations of irrigation with untreated wastewater included an additional study in Mexico and studies conducted in Egypt, China, Cameroon, Burkina Faso, and India. In Mexico, water from a river that receives discharges of untreated domestic wastewater from the city of Chihuahua was used to irrigate two agricultural fields. Irrigation with wastewater-impacted river water stopped on one of the fields 14 years prior to the study but continued on the other. The field continuing to receive wastewater-impacted river water showed a higher number of multidrug-resistant bacteria compared to both the field that no longer receives water from the river and a control field that was rainfed [25]. In a study in Egypt, the incidence of plasmids was $25-50 \%$ higher in isolates from wastewater-irrigated soil than from soils irrigated with canal water, and $>50 \%$ of isolates carrying plasmids were resistant to ampicillin and kanamycin while $>25 \%$ were resistant to tetracycline [28]. A study in China compared agricultural fields, one irrigated with untreated domestic wastewater for over twenty years and a second irrigated with fishpond water, to a field that was not used for cultivation. While the soils irrigated with fishpond water had higher tet and sul relative gene abundances than the wastewater-irrigated fields, ARGs were not detected in the field not used for cultivation [29]. In Cameroon and Burkina Faso, a study researched the impact of irrigation with raw sewage receiving input from homes, hospitals, agriculture, markets, and slaughterhouses compared to non-irrigated soils. Transferable ARGs conferring resistance to trimethoprim, aminoglycosides, beta-lactams, amphenicols, tetracyclines, sulfonamides, macrolides, quinolones, phosphonic antibiotics, and nucleoside antibiotics were $27 \%$ more abundant in wastewater-irrigated soils than in non-irrigated control soils [3]. An additional publication from the same study investigated different AMR mechanisms in both fields, including the presence of genes encoding antibiotic inactivation enzymes, antibiotic target replacement, antibiotic target protection and efflux pumps. The study found the number of ARGs encoding antibiotic inactivation enzymes to be lower in the non-irrigated fields compared to the wastewater-irrigated fields, and the number of ARGs encoding other resistance mechanisms were slightly higher in wastewater-irrigated fields [30].

In four studies in India, fields were irrigated for at least a decade with wastewater that came from factories and domestic sewage. When compared to a groundwater-irrigated field, Pseudomonas spp. isolated from the wastewater-irrigated field had higher resistance towards sulphadiazine, ampicillin, and erythromycin [31]. The other three studies investigated wastewater-irrigated fields in the same area but did not report results from a comparison field. All three studies detected various ARB in wastewater-irrigated fields. These included free-living Azotobacter chroococcum isolates resistant to nitrofurantoin (92\%), polymyxin-B $(86 \%)$, co-trimoxazole $(81 \%)$ and a total of six antibiotics (41\%) [22], bacterial isolates resistant to tetracycline (75\%), doxycycline (58\%), ampicillin (50\%), and nalidixic acid (50\%) [23], and Pseudomonas spp. isolates resistant to cloxacillian (100\%), methicillin (58\%) and a total of four antibiotics (25\%) [24].

\subsection{Irrigation with Treated Wastewater}

Out of thirteen studies focused on treated wastewater, the wastewater effluent was secondary-treated in three studies, a mix of secondary- and tertiary-treated in one study, tertiary-treated in three studies and biologically treated with a wetland system in one study (Table 2). The remaining five studies did not report the extent of treatment. In three studies, the wastewater effluent was diluted prior to irrigation by discharging into an ambient waterbody. Eight studies focused on agricultural fields, four on urban parks and one on a water storage basin recharged with treated wastewater. One study investigated pore water samples while the rest investigated soil. One study did not utilize a comparison field for assessing associations but found ARB in wastewater-irrigated fields. Of the twelve studies that had a comparison site, six found that wastewater irrigation was associated with higher ARB/ARGs in soil while four studies found mixed associations, and in two studies, wastewater irrigation was associated with lower or similar ARB/ARGs in soil compared to irrigation with freshwater, groundwater, and non-irrigated fields. Additional details of the studies are provided in the Supplemental Information (Table S3). 
Table 2. Characteristics of studies on irrigation with treated wastewater.

\begin{tabular}{|c|c|c|c|c|c|c|c|}
\hline Author and Year & Location & WWI Site & WWI Duration & Comparison Site & Target Organism & AMR Mechanism Investigated & Reference \\
\hline Cerqueira et al., 2019 & Spain & $\begin{array}{l}\text { Field irrigated with water from } \\
\text { channel with up to } 92 \% \text { treated } \\
\text { effluent from } 10 \text { wastewater } \\
\text { treatment plants }\end{array}$ & Not reported & $\begin{array}{l}\text { Field irrigated with } \\
\text { ground- and/or } \\
\text { rainwater }\end{array}$ & $\mathrm{N} / \mathrm{A}^{\mathrm{a}}$ & $\begin{array}{l}\text { ARGs: sul1, } b l a_{\mathrm{TEM}}, b l a_{\mathrm{OXA}-58} \\
\text { bla }_{\mathrm{CTX}-\mathrm{M}-32}, \text { mec } \\
\text { Mobile gnenetic elements: intl1 }\end{array}$ & [39] \\
\hline Cerqueira et al., 2019 & Spain & $\begin{array}{l}\text { (1) Field irrigated with water } \\
\text { from channel with up to } 92 \% \\
\text { treated effluent from } 10 \\
\text { wastewater treatment plants } \\
\text { (2) Field irrigated with water } \\
\text { from river that contains }<18 \% \\
\text { treated effluent }\end{array}$ & Not reported & $\begin{array}{l}\text { Field irrigated with } \\
\text { groundwater }\end{array}$ & $\mathrm{N} / \mathrm{A}^{\mathrm{a}}$ & $\begin{array}{l}\text { ARGs: sul1, } b l a_{\mathrm{TEM}}, b l a_{\mathrm{OXA}-58,} \\
\text { bla }_{\mathrm{CTX}-\mathrm{M}-32,} \text { mecA, } q n r S 1, \text { tetM } \\
\text { Mobile genetic elements: } \text { intl1 }\end{array}$ & [40] \\
\hline Chen et al., 2014 & China & $\begin{array}{l}\text { (1) Field irrigated with treated } \\
\text { wastewater directly or from } \\
\text { rivers that receive effluent } \\
\text { (2) Field irrigated with untreated } \\
\text { wastewater until 6-7 years ago, } \\
\text { irrigated with ground- and/or } \\
\text { rainwater since }\end{array}$ & Not reported & Non-irrigated field & $\begin{array}{l}\text { Bacterial isolates } \\
\text { DNA from soil }\end{array}$ & $\begin{array}{l}\text { Resistance against: Oxytetracycline, } \\
\text { Tetracycline, Sulfadiazine, } \\
\text { Sulfamethoxazole } \\
\text { ARGs: } 13 \text { tetracycline resistance genes } \\
(\text { tet } A \text {, tet } B \text {, tet } C \text {, tet } D \text {, tet } E \text {, tet } G \text {, tet } K \text {, } \\
\text { tet } L \text {, tet } M \text {, tet } O \text {, tet } S \text {, tet } Q, \text { tet } X), 3 \\
\text { sulfonamide resistance genes (sul } 1 \text {, } \\
\text { sul } 2, \text { sul } 3 \text { ) }\end{array}$ & {$[32]$} \\
\hline Chigor et al., 2020 & Nigeria & $\begin{array}{l}\text { Earthen pots irrigated with } \\
\text { secondary treated wastewater }\end{array}$ & $\begin{array}{l}\text { Practiced in the } \\
\text { area for }>30 \text { years, } \\
\text { earthen pots } \\
\text { irrigated for } \\
6 \text { weeks }\end{array}$ & None & E. coli isolates & $\begin{array}{l}\text { Resistance against: Amoxicillin, } \\
\text { Ampicillin, Penicillin, Cloxacillin, } \\
\text { Cefuroxime, Streptomycin, } \\
\text { Rifampicin, Metronidazole, } \\
\text { Sulfamethoxazole, Trimethoprim, } \\
\text { Vancomycin, Erythromycin, } \\
\text { Clarithromycin, Chloramphenicol, } \\
\text { Ciprofloxacin, Norfloxacin, } \\
\text { Tetracycline, Imipenem }\end{array}$ & [38] \\
\hline
\end{tabular}


Table 2. Cont.

\begin{tabular}{|c|c|c|c|c|c|c|c|}
\hline Author and Year & Location & WWI Site & WWI Duration & Comparison Site & Target Organism & AMR Mechanism Investigated & Reference \\
\hline Han et al., 2016 & Australia & $\begin{array}{l}\text { Urban park irrigated with } \\
\text { tertiary treated wastewater }\end{array}$ & Not reported & $\begin{array}{l}\text { (1) Urban park } \\
\text { irrigated with } \\
\text { potable water } \\
\text { (2) Pristine soil } \\
\text { from remote } \\
\text { national parks }\end{array}$ & $\mathrm{N} / \mathrm{A}^{\mathrm{a}}$ & $\begin{array}{l}84 \text { ARGs encoding resistance to } \\
\text { aminoglycosides, Classes A, B, C and } \\
\text { D beta-lactam, erythromycin, } \\
\text { quinolones and fluoroquinolones, } \\
\text { macrolide lincosamide } \\
\text { streptogramin_b (MLS_b), multidrug, } \\
\text { tetracycline, vancomycin } \\
\text { Mobile genetic elements: intI1, tnpA } \\
\text { gene of IS6 family transposons }\end{array}$ & [35] \\
\hline Kampouris et al., 2020 & Germany & $\begin{array}{l}\text { Field irrigated with secondary } \\
\text { treated wastewater, sometimes } \\
\text { mixed with digested sludge }\end{array}$ & 50 years & $\begin{array}{l}\text { (1) Period of } \\
\text { irrigation } \\
\text { compared to period } \\
\text { without irrigation } \\
\text { (2) Lab experiment } \\
\text { where soils were } \\
\text { irrigated with } \\
\text { treated wastewater } \\
\text { and freshwater }\end{array}$ & $\mathrm{N} / \mathrm{A}^{\mathrm{a}}$ & $\begin{array}{l}\text { ARGs: sul1, tetM, qnrS, bla } a_{\mathrm{OXA}-58,} \\
\text { bla }_{\mathrm{CTX}-\mathrm{M}-32, b l a_{\mathrm{TEM}}} \\
\text { Mobile genetic elements: intI1 }\end{array}$ & {$[37]$} \\
\hline
\end{tabular}


Table 2. Cont.

\begin{tabular}{|c|c|c|c|c|c|c|c|}
\hline Author and Year & Location & WWI Site & WWI Duration & Comparison Site & Target Organism & AMR Mechanism Investigated & Reference \\
\hline $\begin{array}{l}\text { McLain and } \\
\text { Williams } 2010\end{array}$ & USA & $\begin{array}{l}\text { Soil from water storage basin } \\
\text { recharged with tertiary treated } \\
\text { wastewater }\end{array}$ & $>20$ years & $\begin{array}{l}\text { Soil from water } \\
\text { storage basin } \\
\text { recharged with } \\
\text { groundwater }\end{array}$ & $\begin{array}{l}\text { Enterococcus } \\
\text { isolates }\end{array}$ & $\begin{array}{l}\text { Resistance against: Tigecycline, } \\
\text { Tetracycline, Chloramphenicol, } \\
\text { Daptomycin, Streptomycin, Tylosin } \\
\text { tartrate, Quinupristin/dalfopristin, } \\
\text { Linezolid, Nitrofurantoin, Penicillin, } \\
\text { Kanamycin, Erithromycin, } \\
\text { Ciprofloxacin, Vancomycin, } \\
\text { Lincomycin, Gentamicin }\end{array}$ & {$[44]$} \\
\hline Negreanu et al., 2012 & Israel & $\begin{array}{l}\text { Fields irrigated with secondary } \\
\text { treated wastewater }\end{array}$ & $6,12,15$ years & $\begin{array}{l}\text { Field irrigated with } \\
\text { freshwater, } \\
\text { including aquifer } \\
\text { recharged with } \\
\text { secondary treated } \\
\text { wastewater }\end{array}$ & $\begin{array}{l}\text { Bacterial isolates } \\
\text { DNA from soil }\end{array}$ & $\begin{array}{l}\text { Resistance against: Tetracycline, } \\
\text { Ciprofloxacin, Erythromycin } \\
\text { ARGs: sul1, sul2, ermB, ermF, } \\
\text { tet } O, \text { qnrA }\end{array}$ & [41] \\
\hline Palacios et al., 2017 & Mexico & $\begin{array}{l}\text { Recreational parks irrigated } \\
\text { with tertiary treated wastewater }\end{array}$ & Not reported & $\begin{array}{l}\text { Distance from } \\
\text { WWTP }\end{array}$ & Bacterial isolates & $\begin{array}{l}\text { Resistance against: Ampicillin, } \\
\text { Riphampicin, Chloramphenicol, } \\
\text { Ciprofloxacin, Gentamicin, } \\
\text { Trimethoprim-sulphametoxazole }\end{array}$ & [36] \\
\hline Troiano et al., 2018 & Israel & $\begin{array}{l}\text { Field irrigated with greywater } \\
\text { treated by recirculating vertical } \\
\text { flow constructed wetland }\end{array}$ & $>7$ years & $\begin{array}{l}\text { Field irrigated with } \\
\text { freshwater }\end{array}$ & Bacterial isolates & $\begin{array}{l}\text { Resistance against: Tetracycline, } \\
\text { Amoxicillin, Ciprofloxacin, } \\
\text { Kanamycin } \\
\text { ARGs: Beta-lactamase genes }\left(\text { bla } a_{\mathrm{TEM}},\right. \\
\left.\text { bla }_{\mathrm{CTXM}-32}, \text { bla }_{\mathrm{SHV}}, \text { bla }{ }_{\mathrm{OXA}-2}, b l a_{\mathrm{OXA} 10}\right) \\
\text { tetracycline resistance genes }(\text { tet } 39, \\
\text { tet } A \text {, tet } B \text { tet } M \text {, tet } Q, \text { tet } W)\end{array}$ & [43] \\
\hline Wang et al., 2014 & China & $\begin{array}{l}\text { Public parks irrigated with } \\
\text { treated wastewater }\end{array}$ & Not reported & $\begin{array}{l}\text { Pristine remote } \\
\text { parks }\end{array}$ & $\mathrm{N} / \mathrm{A}^{\mathrm{a}}$ & $\begin{array}{l}\text { ARGs: } 15 \text { tetracycline resistance } \\
\text { genes, } 4 \text { beta-lactamase genes, } 3 \\
\text { quinolone resistance genes } \\
\text { Mobile genetic elements: intl1 }\end{array}$ & {$[34]$} \\
\hline Wang et al., 2014 & China & $\begin{array}{l}\text { Urban parks irrigated with } \\
\text { treated wastewater in seven } \\
\text { cities }\end{array}$ & $3-12$ years & $\begin{array}{l}\text { Urban parks not } \\
\text { irrigated with } \\
\text { reclaimed water in } \\
\text { same cities }\end{array}$ & $\mathrm{N} / \mathrm{A}^{\mathrm{a}}$ & $\begin{array}{l}\text { ARGS: } 285 \text { different ARGs } \\
\text { Mobile genetic elements: } 9 \\
\text { transposase genes }\end{array}$ & [33] \\
\hline
\end{tabular}

WWI: Wastewater irrigation; AMR: Antimicrobial resistance; ARG: Antimicrobial resistance gene. ${ }^{\text {a }}$ No specific target organism, DNA extracted directly from soil. 
The six studies that found a positive association were conducted in China, Australia, Mexico and Germany. In China, a study compared a field irrigated with treated wastewater (either directly or indirectly from a river receiving discharge), a field that was irrigated with untreated wastewater until 6-7 years ago and with rain- and groundwater since then, and a third field that is non-irrigated. The study found that the relative abundance of sulfadiazineresistant bacteria was highest in the field previously irrigated with untreated wastewater, with no other differences in the relative abundance of ARBs between the fields. The relative abundance of tet $A$, tet $C$, tet $E$, tet $G$, tetS, sul 1 and sul3 genes as well as the sum of the relative abundances of tet and sul genes were significantly higher in currently and previously wastewater-irrigated soils than in non-irrigated soils [32]. There was no difference in relative abundance of ARGs between currently and previously wastewater-irrigated soils. Two other studies in China focused on public parks irrigated with reclaimed wastewater but did not report the type and degree of treatment. In one of these studies, there was a higher diversity and abundance of ARGs encoding beta-lactam, FCA (fluoroquinolone, quinolone, florfenicol, chloramphenicol, amphenicol), and aminoglycoside resistance in soil from urban parks irrigated with reclaimed wastewater than in control soils from urban parks in the same cities not irrigated with wastewater [33]. The abundance of ARGs was 99-8655 times higher in wastewater-irrigated parks while the abundance of transposase genes was up to 2959 times higher compared to control soils. The second study also found higher diversity and absolute abundance of sul1 genes $\left(1.69 \times 10^{8}\right.$ copies per g dry soil) and intl1 genes $\left(7.62 \times 10^{7}\right.$ copies per g dry soil) in soil irrigated with reclaimed water than in pristine soils from national parks $\left(9.08 \times 10^{7}\right.$ and $2.61 \times 10^{7}$ copies per g dry soil) [34].

A similar study in Australia compared urban parks irrigated with tertiary-treated wastewater, urban parks irrigated with potable water and remote national parks. Wastewaterirrigated parks had a higher number of different ARGs than both other sites. The abundance of ARGs in soil from wastewater-irrigated parks, conferring resistance to all major classes of antibiotics, except for erythromycin and vancomycin, was 815-4300 times higher than soil from a national park. Urban parks without wastewater irrigation, on the other hand, had 150-1240 times higher prevalence of ARGs than soil from the national park [35]. There was no difference in the relative abundance of $i n t l 1$ and $\operatorname{tnp} A$ genes between sites. In a study in Mexico, soil from recreational parks irrigated with tertiary-treated wastewater had a higher number of multi-drug resistant bacteria in parks closer to the wastewater treatment plant compared to parks further away [36].

Finally, a study in Germany compared ARGs in subsoil pore-water in fields irrigated with secondary-treated wastewater during periods of different irrigation intensity and a period with no irrigation. The relative abundance of sul, tet, qnr, bla and intl1 genes was higher during high-intensity irrigation compared to the irrigation break, and the relative abundance of several ARGs increased with increasing irrigation intensity [37]. A lab study was set up to replicate the field study and confirmed that the relative abundance of ARGs was higher in soils irrigated with treated wastewater versus freshwater [37]. Additionally, a study in Nigeria investigated soil irrigated with secondary-treated wastewater. While the study did not use a comparison site, $100 \%$ of $E$. coli isolates from wastewater-irrigated soils were resistant to $\geq 5$ antibiotics [38].

The six studies that found mixed or negative associations between wastewater irrigation and ARB/ARGs in soil were conducted in Spain, Israel and the US. Two studies in Spain investigated fields irrigated with wastewater from a channel that received up to $92 \%$ effluent from 10 wastewater treatment plants versus fields irrigated with rain- or groundwater. In the first study, the relative abundance of $t e t \mathrm{M}$, mec $\mathrm{A}$, qnrS1 and bla $_{\mathrm{OXA}-58}$ genes was higher in wastewater-irrigated fields, but the relative abundance of bla $a_{\mathrm{CTX}-\mathrm{M}-32}$ was higher in the groundwater-irrigated areas [39]. The second study also investigated a third field irrigated with wastewater-impacted river water, where wastewater effluent made up $<18 \%$ of the water flow. The abundance of intl1 genes was higher in soil irrigated with groundwater but the highest abundance of $b l a_{\text {TEM }}$ was found in the soils irrigated 
with river water containing $<18 \%$ wastewater effluent, while the abundance of qnrS1 genes was higher in both wastewater-irrigated fields [40].

In Israel, a study compared fields irrigated with secondary-treated wastewater to fields irrigated with freshwater, including groundwater from an aquifer recharged with secondary-treated wastewater. The relative abundance of ARB was similar or higher in the freshwater-irrigated soils. Absolute gene copy numbers for ARGs tested (sul1, sul2, ermB, ermF, tet $O$, and $q n r A$ ) were similar or higher in the freshwater-irrigated soils at three out of four study sites while they were higher in wastewater-irrigated soils at the remaining site. Similarly, the relative abundance of ARGs was higher in the freshwater-irrigated soils at three sites and higher in wastewater-irrigated soils at the fourth site [41]. Notably, one of the comparison sites in this study was irrigated with groundwater from an aquifer that is recharged with secondary-treated wastewater. In a second study in Israel, commercial agriculture fields irrigated with secondary- and tertiary-treated wastewater were compared to fields irrigated with surface water, groundwater, or desalinated water. The study also examined an experimental orchard and lysimeters irrigated with tertiary-treated wastewater and freshwater. Wastewater-irrigated soil in lysimeters had higher relative and absolute abundance of intl1 genes compared to freshwater-irrigated lysimeters. However, almost all ARGs were below detection limits in all tested soils, even after irrigation with treated wastewater [42]. A third study in Israel compared soils irrigated with greywater treated by constructed wetlands to soils irrigated with freshwater, with no difference in the abundance of tetracycline-resistant bacteria between the two types of soils [43].

Finally, a study in the U.S. investigated Enterococcus from sediments of a basin recharged with tertiary-treated wastewater for more than 20 years and compared it to enterococci isolated from soils and sediments in a groundwater-filled pond. A higher proportion of bacteria isolated from the groundwater-filled pond was resistant to 4-6 antibiotics $(25 \%)$ than bacteria from the wastewater-recharged pond (9\%), and a smaller proportion of bacteria from the groundwater-filled pond was susceptible to all antibiotics tested $(7 \%)$ than bacteria from the wastewater-recharged pond (36\%) [44].

\subsection{Effect of Other Environmental Factors}

Other environmental factors besides wastewater irrigation had impacts on the abundance and diversity of AMR in soil. Multiple studies noted the impact of soil moisture, precipitation, temperature, $\mathrm{pH}$ and soil depth. A study in Israel found soil moisture had a significant positive correlation with bacterial resistance to tetracycline and ciprofloxacin [41]. A study in Germany found that the relative abundance of sul1 and plasmid-borne $q n r S$ genes in subsoil pore water increased with increasing temperature, and the relative abundance of sull genes was positively correlated with precipitation, but there was no correlation between ARGs and humidity [37]. Similarly, in Mexico, the prevalence of ARB in wastewater-irrigated soils was lower during the dry sampling period compared to the rainy period [36]. Evidence on the effect of soil $\mathrm{pH}$ was mixed. A study in Australia found ARG abundance in soil to increase with soil $\mathrm{pH}$ [35] while conversely, in another study, higher soil $\mathrm{pH}$ was negatively correlated with the abundance of ARGs and the intl1 gene [34]. In a study in Mexico, there was no association between soil $\mathrm{pH}$ and ARG abundance [21]. Most studies investigated top soils $(0-30 \mathrm{~cm}$ depth) and some studies assessed the effect of soil depth on ARB/ARGs. In Mexico, the prevalence of multi-resistant bacteria was not affected by soil depth, comparing samples collected at 0-15, 15-30 and 30-50 cm depth [25]. Similarly, In China, the relative abundance of ARGs was not significantly different between soil depths of 0-10 cm and 10-20 cm [29]. Aggregation of agricultural soil may also play a role in the dissemination of AMR in wastewater-irrigated fields. A study in China found no difference in ARG abundance between rhizosphere, non-rhizosphere and wetland samples [34]. In a study in Mexico, untreated domestic wastewater used to irrigate soil cores was dyed before irrigation to visualize water flow paths. The dye stained a greater volume and deeper in the soil cores collected from wastewater-irrigated fields $(80 \%)$ than those in the more compacted rainfed fields where the dyed water followed the 
root system rather than penetrating a larger area of the soil core (50\%). The abundance of sull and sul2 genes was higher in stained soil compartments along the flow path than in unstained compartments, suggesting that water flow paths could be an area of concern with high levels of resistance genes [27].

\section{Discussion}

This review summarizes results from 26 studies on the impact of wastewater irrigation on the prevalence and abundance of ARB and ARGs in soil and water. Our review indicates that an important determinant of the presence of AMR in wastewater-irrigated soil is whether the wastewater used for irrigation was treated. We found evidence of a positive relationship between irrigation with untreated wastewater and both the presence and abundance of ARB/ARGs in soil, where nine out of ten studies that had a comparison group (e.g., fields irrigated with freshwater) showed an increase in ARB and ARGs in wastewater-irrigated soils. In contrast, studies that investigated irrigation with treated wastewater had heterogeneous findings. Out of the twelve studies in this category that had a comparison group, wastewater irrigation was associated with more abundant ARB/ARGs in soil in six studies, while the remaining six studies found mixed or negative associations.

Our review also revealed that studies examining ARB and ARGs in water bodies due to wastewater irrigation are currently limited. Only one study in our review studied sub-pore water, and we identified no studies investigating AMR in underlying groundwater aquifers or surface water bodies adjacent to wastewater-irrigated fields. Wastewater irrigation has been associated with the detection of pathogens, nitrates, and antibiotics in surface- and groundwaters [45]. Future research should investigate whether ARB/ARGs are detected in waters impacted by wastewater irrigation.

\subsection{Differences in Wastewater Treatment}

Our findings highlight the need to further investigate the drivers of heterogeneity to identify settings and factors that modify the risk associated with wastewater irrigation. Notably, the studies focused on untreated wastewater exclusively came from middle and low-income countries while eight out of thirteen studies on treated wastewater came from high-income countries. AMR carriage is significantly higher in low-income countries, which has been attributed to unregulated antibiotic use and poor sanitary conditions $[46,47]$. Therefore, wastewater used for irrigation in low-income countries is more likely to contain ARB/ARGs. The extent and effectiveness of wastewater treatment also differs between high- and low-income countries. The six studies that investigated irrigation with treated wastewater and found mixed or negative effects on ARB/ARGs in soil were conducted in high-income countries with presumably effective and well-operating wastewater treatment systems, while the majority of the studies that found an increase in ARB/ARGs in soil from irrigation with treated wastewater came from low-income countries. Therefore, differences in ARB/ARG loads in wastewater and removal efficiency for ARB/ARGs in wastewater treatment plants between high- and low-income countries could explain why studies on irrigation with untreated wastewater found an increase in AMR in soil while studies on irrigation with treated wastewater had heterogeneous findings.

Differences in the types of wastewater treatment steps employed would also be expected to affect the presence of antibiotics, ARB, and ARGs in the treated effluent and consequently the impact on soils. However, studies in our review that focused on secondary vs. tertiary-treated wastewater had similarly mixed findings. Among the three studies that investigated tertiary-treated wastewater, two found a positive association between wastewater irrigation and ARB [36] and ARGs [35] while the third found a negative association with ARB [44]. Whether or not the wastewater or treated effluent was diluted via discharge into an ambient waterbody prior to irrigation also did not appear to influence the effect of wastewater irrigation on ARB/ARGs in soil. 


\subsection{Duration of Irrigation}

It is possible that ARB from irrigation with wastewater could take several years to accumulate in soil [48]. The duration of wastewater irrigation prior to sampling varied immensely (1.5 to 100 years) across the studies in our review, and nine out of 26 studies did not report the duration. Based on studies in Mezquital Valley, duration of irrigation has implications for the dissemination of ARB/ARGs within wastewater-irrigated soils $[19,21]$. Therefore, mixed findings between studies could be due to differences in the duration of wastewater irrigation. In addition, when ARB and ARGs are detected in wastewaterirrigated soils, it is unknown whether and how long they persist, either through the survival of the host bacteria or as free naked DNA [18]. Few studies in our review reported the time elapsed between the last episode of wastewater irrigation and collection of samples, and in most studies, soils appeared to be sampled concurrently with ongoing wastewater irrigation. Three studies investigated fields where wastewater irrigation was discontinued and had mixed findings. In Spain, the field currently irrigated with untreated wastewater had the highest abundance of multi-resistant bacteria while the field previously irrigated with untreated wastewater and the rainfed control field had similar abundance of multiresistant bacteria [25]. In a study in Germany, the relative abundance of ARGs was higher during periods with active irrigation compared to after a 4-month irrigation break [37]. In contrast, in China, there was no difference in the relative abundance of ARB and ARGs between fields currently vs. previously irrigated with wastewater [32]. Other facets of wastewater irrigation, such as the origin of wastewater, and the intensity, frequency and volume of irrigation can also modify the effect of wastewater irrigation on AMR in soil; these factors were only partially reported by studies in our review.

\subsection{Pre-Existing AMR in Soil}

Mechanisms for AMR naturally exist in native soil communities [49]. When determining the impact of wastewater irrigation on ARB and ARGs, it can be difficult to assess the respective contribution of wastewater due to the natural bacteria and resistance already present in the soil [41]. DNA can exist in soil for long periods of time so when researchers use molecular methods of detection, such as polymerase chain reaction (PCR), they might detect pre-existing native bacteria that have been in the soil for many years [12]. Many studies in our review included soil samples that were not wastewater-irrigated, allowing a comparison to isolate the impact of wastewater. However, comparison soils can also be contaminated with AMR elements if they are close to wastewater-irrigated sites (via aerosols and dust) or if they have received soil amendment with manure or biosolids [19]. Only a few studies in our review used "pristine" comparison soils from remote areas with less anthropogenic activity.

\subsection{Analytic Methods and Detection Limits}

Selection of which ARB/ARGs were investigated can also lead to heterogeneous findings across studies. It is also important to note that due to limits of detection and quantification, PCR can fail to detect or quantify ARGs that are present in low levels, which may still have a biological impact [18]. Levels of ARGs can be expressed as a gene ratio, comparing the gene copy numbers of the ARG to those of a common gene such as $16 \mathrm{~S}$ rRNA. These ratios are used to define the relative abundance of the ARG and can be too low to be interpreted or compared between samples [18].

\subsection{Environmental Factors}

Finally, it is important to include details of soil properties and environmental characteristics in future studies. Multiple studies in our review indicated associations between the abundance of ARGs and soil $\mathrm{pH}$, soil moisture, precipitation and temperature. Additionally, the fate and transport of antibiotics in soil and the DNA extraction efficiency from soil samples can vary with varying soil properties [41]. Reporting soil and environmental 
characteristics in future studies could help identify factors that may modify the effect of wastewater irrigation on the presence and abundance of ARB/ARGs in soil.

\subsection{Potential Human Health Risks}

Studies have attempted to estimate the human health risk from exposure to antibiotics through wastewater irrigation [15,50], but the health effects of potential exposure to $\mathrm{ARB}$ and/or ARGs due to wastewater irrigation are unclear [18,51]. Individuals can be exposed to these through contact with soil or consumption of crops that have taken up ARB / ARGs from wastewater-irrigated soil, potentially leading to gut colonization with resistant bacteria. However, environment-to-human transmission of AMR remains poorly understood [52]. Studies using advanced molecular techniques such as whole genome sequencing have shown genetic overlap between ARB isolated from humans, animals and the farm environment, suggesting transmission between these reservoirs and hosts through farming practices such as soil amendment with manure $[53,54]$. Similar risks could exist for farmers as well as consumers due to environment-to-human transmission and spread of AMR when untreated wastewater is used for irrigation [55]. Low-income countries, where most wastewater remains untreated and is also more likely to contain ARB/ARGs due to high community carriage rates, are a particularly high-risk setting for further emergence and spread of AMR via wastewater irrigation. Novel resistance mechanisms that emerge in such hotspots have been shown to quickly spread globally [56,57]. Irrigation with untreated wastewater could therefore pose risks beyond the countries where it is practiced.

\section{Conclusions}

Given scarce water resources, climate change and population growth, wastewater irrigation is increasingly common, in both low- and high-income countries. Through a systematic review and synthesis of the available literature, we demonstrate the diverse impact that domestic wastewater irrigation can have on the presence of AMR in soil. Our findings indicate a clear relationship between untreated wastewater irrigation and increasing prevalence and abundance of ARB and ARGs in soil. While there are no studies on the magnitude of human health risks associated with exposure to AMR via irrigation with untreated wastewater, our findings warrant precautionary action by field workers, their families, and consumers, particularly in low-income countries where use of raw sewage for irrigation is common. Studies should also investigate whether irrigation with untreated wastewater leads to contamination of adjacent water sources with ARB and ARGs. In our review, the evidence on whether irrigation with treated wastewater increases the prevalence and abundance of AMR in soil was mixed. Future research should explore factors that can explain the heterogeneity in the effect of irrigation with treated wastewater on ARB and ARGs in soil, such as the extent of wastewater treatment and the intensity of irrigation, to inform guidelines on wastewater reuse for irrigation.

Supplementary Materials: The following are available online at https:/ /www.mdpi.com/article/10 .3390/ijerph182111046/s1, Table S1: Search terms, Text S1: PubMed search string, Table S2: PRISMA checklist, Table S3: Characteristics of studies included in review.

Author Contributions: Conceptualization, S.S. and A.E.; methodology, S.S. and A.E.; investigation, S.S. and C.N.; data curation, S.S.; writing - original draft preparation, S.S. and A.E.; writing-review and editing, S.S, C.N., G.A., S.T. and A.E.; visualization, S.S.; supervision, A.E.; project administration, S.S. and A.E. All authors have read and agreed to the published version of the manuscript.

Funding: This research received no external funding.

Institutional Review Board Statement: Not applicable.

Informed Consent Statement: Not applicable.

Conflicts of Interest: The authors declare no conflict of interest. 


\section{References}

1. Wallace, J.S. Increasing agricultural water use efficiency to meet future food production. Agric. Ecosyst. Environ. 2000, 82, 105-119. [CrossRef]

2. Odegard, I.Y.R.; Voet, E. van der The future of food-Scenarios and the effect on natural resource use in agriculture in 2050. Ecol. Econ. 2014, 97, 51-59. [CrossRef]

3. Bougnom, B.P.; Thiele-Bruhn, S.; Ricci, V.; Zongo, C.; Piddock, L.J.V. Raw wastewater irrigation for urban agriculture in three African cities increases the abundance of transferable antibiotic resistance genes in soil, including those encoding extended spectrum $\beta$-lactamases (ESBLs). Sci. Total Environ. 2020, 698, 134201. [CrossRef]

4. Saldias, C.; Speelman, S.; Huylenbroeck, G.V.; Vink, N. Understanding farmers' preferences for wastewater reuse frameworks in agricultural irrigation: Lessons from a choice experiment in the western cape, South Africa. Water SA 2016, 42, 26-37. [CrossRef]

5. Sato, T.; Qadir, M.; Yamamoto, S.; Endo, T.; Zahoor, A. Global, regional, and country level need for data on wastewater generation, treatment, and use. Agric. Water Manag. 2013, 130, 1-13. [CrossRef]

6. Wang, Z.; Li, J.; Li, Y. Using Reclaimed Water for Agricultural and Landscape Irrigation in China: A Review. Irrig. Drain. 2017, 66, 672-686. [CrossRef]

7. Pop, C.-E.; Draga, S.; Măciucă, R.; Niță, R.; Crăciun, N.; Wolff, R. Bisphenol A Effects in Aqueous Environment on Lemna minor. Processes 2021, 9, 1512. [CrossRef]

8. Dickin, S.K.; Schuster-Wallace, C.J.; Qadir, M.; Pizzacalla, K. A review of health risks and pathways for exposure to wastewater Use in Agriculture. Environ. Health Perspect. 2016, 124, 900-909. [CrossRef] [PubMed]

9. Liu, J.L.; Wong, M.H. Pharmaceuticals and personal care products (PPCPs): A review on environmental contamination in China. Environ. Int. 2013, 59, 208-224. [CrossRef] [PubMed]

10. Nnadozie, C.F.; Kumari, S.; Bux, F. Status of pathogens, antibiotic resistance genes and antibiotic residues in wastewater treatment systems. Rev. Environ. Sci. Biotechnol. 2017, 16, 491-515. [CrossRef]

11. Gatica, J.; Cytryn, E. Impact of treated wastewater irrigation on antibiotic resistance in the soil microbiome. Environ. Sci. Pollut. Res. 2013, 20, 3529-3538. [CrossRef]

12. Christou, A.; Agüera, A.; Bayona, J.M.; Cytryn, E.; Fotopoulos, V.; Lambropoulou, D.; Manaia, C.M.; Michael, C.; Revitt, M.; Schröder, P.; et al. The potential implications of reclaimed wastewater reuse for irrigation on the agricultural environment: The knowns and unknowns of the fate of antibiotics and antibiotic resistant bacteria and resistance genes-A review. Water Res. 2017, 123, 448-467. [CrossRef]

13. Sorinolu, A.J.; Tyagi, N.; Kumar, A.; Munir, M. Antibiotic resistance development and human health risks during wastewater reuse and biosolids application in agriculture. Chemosphere 2021, 265, 129032. [CrossRef] [PubMed]

14. Ashbolt, N.J.; Amézquita, A.; Backhaus, T.; Borriello, P.; Brandt, K.K.; Collignon, P.; Coors, A.; Finley, R.; Gaze, W.H.; Heberer, T.; et al. Human health risk assessment (HHRA) for environmental development and transfer of antibiotic resistance. Environ. Health Perspect. 2013, 121, 993-1001. [CrossRef]

15. Piña, B.; Bayona, J.M.; Christou, A.; Fatta-Kassinos, D.; Guillon, E.; Lambropoulou, D.; Michael, C.; Polesel, F.; Sayen, S. On the contribution of reclaimed wastewater irrigation to the potential exposure of humans to antibiotics, antibiotic resistant bacteria and antibiotic resistance genes-NEREUS COST Action ES1403 position paper. J. Environ. Chem. Eng. 2020, 8, 102131. [CrossRef]

16. Oztekin, T.; Brown, L.C.; Holdsworth, P.M.; Kurunc, A.; Rector, D. Evaluating drainage design parameters forwastewater irrigation applications to minimize impact on surfacewaters. Appl. Eng. Agric. 1999, 99, 449-455. [CrossRef]

17. Santos, L.H.M.L.M.; Araújo, A.N.; Fachini, A.; Pena, A.; Delerue-Matos, C.; Montenegro, M.C.B.S.M. Ecotoxicological aspects related to the presence of pharmaceuticals in the aquatic environment. J. Hazard. Mater. 2010, 175, 45-95. [CrossRef]

18. Becerra-Castro, C.; Lopes, A.R.; Vaz-Moreira, I.; Silva, E.F.; Manaia, C.M.; Nunes, O.C. Wastewater reuse in irrigation: A microbiological perspective on implications in soil fertility and human and environmental health. Environ. Int. 2015, 75, 117-135. [CrossRef] [PubMed]

19. Dalkmann, P.; Broszat, M.; Siebe, C.; Willaschek, E.; Sakinc, T.; Huebner, J.; Amelung, W.; Grohmann, E.; Siemens, J. Accumulation of pharmaceuticals, enterococcus, and resistance genes in soils irrigated with wastewater for zero to 100 years in central Mexico. PLoS ONE 2012, 7, e45397. [CrossRef]

20. Broszat, M.; Nacke, H.; Blasi, R.; Siebe, C.; Huebner, J.; Daniel, R.; Grohmanna, E. Wastewater irrigation increases the abundance of potentially harmful Gammaproteobacteria in soils in Mezquital Valley, Mexico. Appl. Environ. Microbiol. 2014, 80, 5282-5291. [CrossRef]

21. Jechalke, S.; Broszat, M.; Lang, F.; Siebe, C.; Smalla, K.; Grohmann, E. Effects of 100 years wastewater irrigation on resistance genes, class 1 integrons and IncP-1 plasmids in Mexican soil. Front. Microbiol. 2015, 6, 163. [CrossRef]

22. Aleem, A.; Isar, J.; Malik, A. Impact of long-term application of industrial wastewater on the emergence of resistance traits in Azotobacter chroococcum isolated from rhizospheric soil. Bioresour. Technol. 2003, 86, 7-13. [CrossRef]

23. Ansari, M.I.; Grohmann, E.; Malik, A. Conjugative plasmids in multi-resistant bacterial isolates from Indian soil. J. Appl. Microbiol. 2008, 104, 1774-1781. [CrossRef] [PubMed]

24. Shafiani, S.; Malik, A. Tolerance of pesticides and antibiotic resistance in bacteria isolated from wastewater-irrigated soil. World J. Microbiol. Biotechnol. 2003, 19, 897-901. [CrossRef] 
25. Palacios, O.A.; Contreras, C.A.; Muñoz-Castellanos, L.N.; González-Rangel, M.O.; Rubio-Arias, H.; Palacios-Espinosa, A.; Nevárez-Moorillón, G.V. Monitoring of indicator and multidrug resistant bacteria in agricultural soils under different irrigation patterns. Agric. Water Manag. 2017, 184, 19-27. [CrossRef]

26. Lesser, L.E.; Mora, A.; Moreau, C.; Mahlknecht, J.; Hernández-Antonio, A.; Ramírez, A.I.; Barrios-Piña, H. Survey of 218 organic contaminants in groundwater derived from the world's largest untreated wastewater irrigation system: Mezquital Valley, Mexico. Chemosphere 2018, 198, 510-521. [CrossRef] [PubMed]

27. Lüneberg, K.; Prado, B.; Broszat, M.; Dalkmann, P.; Díaz, D.; Huebner, J.; Amelung, W.; López-Vidal, Y.; Siemens, J.; Grohmann, E.; et al. Water flow paths are hotspots for the dissemination of antibiotic resistance in soil. Chemosphere 2018, 193, 1198-1206. [CrossRef] [PubMed]

28. Bahig, A.E.; Aly, E.A.; Khaled, A.A.; Amel, K.A. Isolation, characterization and application of bacterial population from agricultural soil at Sohag Province, Egypt. Malays. J. Microbiol. 2008, 4, 42-50. [CrossRef]

29. Pan, M.; Chu, L.M. Occurrence of antibiotics and antibiotic resistance genes in soils from wastewater irrigation areas in the Pearl River Delta region, southern China. Sci. Total Environ. 2018, 624, 145-152. [CrossRef]

30. Bougnom, B.P.; Thiele-Bruhn, S.; Ricci, V.; Zongo, C.; Piddock, L.J.V. High-throughput sequencing data and antibiotic resistance mechanisms of soil microbial communities in non-irrigated and irrigated soils with raw sewage in African cities. Data Brief 2019, 27, 104638. [CrossRef]

31. Malik, A.; Aleem, A. Incidence of metal and antibiotic resistance in Pseudomonas spp. from the river water, agricultural soil irrigated with wastewater and groundwater. Environ. Monit. Assess. 2011, 178, 293-308. [CrossRef]

32. Chen, C.; Li, J.; Chen, P.; Ding, R.; Zhang, P.; Li, X. Occurrence of antibiotics and antibiotic resistances in soils from wastewater irrigation areas in Beijing and Tianjin, China. Environ. Pollut. 2014, 193, 94-101. [CrossRef]

33. Wang, F.H.; Qiao, M.; Su, J.Q.; Chen, Z.; Zhou, X.; Zhu, Y.G. High throughput profiling of antibiotic resistance genes in urban park soils with reclaimed water irrigation. Environ. Sci. Technol. 2014, 48, 9079-9085. [CrossRef]

34. Wang, F.H.; Qiao, M.; Lv, Z.E.; Guo, G.X.; Jia, Y.; Su, Y.H.; Zhu, Y.G. Impact of reclaimed water irrigation on antibiotic resistance in public parks, Beijing, China. Environ. Pollut. 2014, 184, 247-253. [CrossRef]

35. Han, X.M.; Hu, H.W.; Shi, X.Z.; Wang, J.T.; Han, L.L.; Chen, D.; He, J.Z. Impacts of reclaimed water irrigation on soil antibiotic resistome in urban parks of Victoria, Australia. Environ. Pollut. 2016, 211, 48-57. [CrossRef]

36. Palacios, O.A.; Serna, F.J.Z.-D.; de la Ballinas-Casarrubias, M.; Espino-Valdés, M.S.; Nevárez-Moorillón, G.V. Microbiological impact of the use of reclaimed wastewater in recreational parks. Int. J. Environ. Res. Public Health 2017, 14, 1009.[CrossRef] [PubMed]

37. Kampouris, I.D.; Klümper, U.; Agrawal, S.; Orschler, L.; Cacace, D.; Kunze, S.; Berendonk, T.U. Treated wastewater irrigation promotes the spread of antibiotic resistance into subsoil pore-water. Environ. Int. 2021, 146, 106190. [CrossRef] [PubMed]

38. Chigor, V.; Ibangha, I.A.; Chigor, C.; Titilawo, Y. Treated wastewater used in fresh produce irrigation in Nsukka, Southeast Nigeria is a reservoir of enterotoxigenic and multidrug-resistant Escherichia coli. Heliyon 2020, 6, e03780. [CrossRef] [PubMed]

39. Cerqueira, F.; Matamoros, V.; Bayona, J.; Piña, B. Antibiotic resistance genes distribution in microbiomes from the soil-plant-fruit continuum in commercial Lycopersicon esculentum fields under different agricultural practices. Sci. Total Environ. 2019, 652, 660-670. [CrossRef] [PubMed]

40. Cerqueira, F.; Matamoros, V.; Bayona, J.; Elsinga, G.; Hornstra, L.M.; Piña, B. Distribution of antibiotic resistance genes in soils and crops. A field study in legume plants (Vicia faba L.) grown under different watering regimes. Environ. Res. 2019, 170, 16-25. [CrossRef]

41. Negreanu, Y.; Pasternak, Z.; Jurkevitch, E.; Cytryn, E. Impact of treated wastewater irrigation on antibiotic resistance in agricultural soils. Environ. Sci. Technol. 2012, 46, 4800-4808. [CrossRef] [PubMed]

42. Marano, R.B.M.; Zolti, A.; Jurkevitch, E.; Cytryn, E. Antibiotic resistance and class 1 integron gene dynamics along effluent, reclaimed wastewater irrigated soil, crop continua: Elucidating potential risks and ecological constraints. Water Res. 2019, 164, 114906. [CrossRef] [PubMed]

43. Troiano, E.; Beneduce, L.; Gross, A.; Ronen, Z. Antibiotic-resistant bacteria in greywater and greywater-irrigated soils. Front. Microbiol. 2018, 9, 2666. [CrossRef] [PubMed]

44. Mclain, J.E.T.; Williams, C.F. Development of Antibiotic Resistance in Bacteria of Soils Irrigated with Reclaimed Wastewater. In Proceedings of the 5th National Decennial Irrigation Conference, Phoenix, AZ, USA, 5-8 December 2010; pp. 1-10.

45. Dcosta, V.M.; King, C.E.; Kalan, L.; Morar, M.; Sung, W.W.L.; Schwarz, C.; Froese, D.; Zazula, G.; Calmels, F.; Debruyne, R.; et al. Antibiotic resistance is ancient. Nature 2011, 477, 457-461. [CrossRef]

46. Ungureanu, N.; Vlăduț, V.; Voicu, G. Water scarcity and wastewater reuse in crop irrigation. Sustainability 2020, 12, 9055. [CrossRef]

47. Collignon, P.; Beggs, J.J.; Walsh, T.R.; Gandra, S.; Laxminarayan, R. Articles Anthropological and socioeconomic factors contributing to global antimicrobial resistance: A univariate and multivariable analysis. Lancet Planet. Health 2018, 2, e398-e405. [CrossRef]

48. Laxminarayan, R.; Duse, A.; Wattal, C.; Zaidi, A.K.M.; Wertheim, H.F.L.; Sumpradit, N.; Vlieghe, E.; Hara, G.L.; Gould, I.M.; Goossens, H.; et al. Antibiotic resistance-the need for global solutions. Lancet Infect. Dis. 2013, 13, 1057-1098. [CrossRef]

49. Raynaud, X.; Nunan, N. Spatial ecology of bacteria at the microscale in soil. PLoS ONE 2014, 9, e87217. [CrossRef]

50. Adegoke, A.A.; Amoah, I.D.; Stenström, T.A.; Verbyla, M.E.; Mihelcic, J.R. Epidemiological evidence and health risks associated with agricultural reuse of partially treated and untreated wastewater: A review. Front. Public Health 2018, 6, 337. [CrossRef] 
51. Graham, D.W.; Bergeron, G.; Bourassa, M.W.; Dickson, J.; Gomes, F.; Howe, A.; Kahn, L.H.; Morley, P.S.; Scott, H.M.; Simjee, S.; et al. Complexities in understanding antimicrobial resistance across domesticated animal, human, and environmental systems. Ann. N. Y. Acad. Sci. 2019, 1441, 17-30. [CrossRef] [PubMed]

52. Manaia, C.M. Assessing the Risk of Antibiotic Resistance Transmission from the Environment to Humans: Non-Direct Proportionality between Abundance and Risk. Trends Microbiol. 2017, 25, 173-181. [CrossRef] [PubMed]

53. Keelara, S.; Scott, H.M.; Morrow, W.M.; Gebreyes, W.A.; Correa, M.; Nayak, R.; Stefanova, R.; Thakur, S. Longitudinal study of distributions of similar antimicrobial-resistant Salmonella serovars in pigs and their environment in two distinct swine production systems. Appl. Environ. Microbiol. 2013, 79, 5167-5178. [CrossRef]

54. Pornsukarom, S.; Van Vliet, A.H.M.; Thakur, S. Whole genome sequencing analysis of multiple Salmonella serovars provides insights into phylogenetic relatedness, antimicrobial resistance, and virulence markers across humans, food animals and agriculture environmental sources. BMC Genom. 2018, 19, 801. [CrossRef]

55. Bougnom, B.P.; Piddock, L.J.V. Wastewater for Urban Agriculture: A Significant Factor in Dissemination of Antibiotic Resistance. Environ. Sci. Technol. 2017, 51, 5863-5864. [CrossRef]

56. Johnson, A.P.; Woodford, N. Global spread of antibiotic resistance: The example of New Delhi metallo- $\beta$-lactamase (NDM)mediated carbapenem resistance. J. Med. Microbiol. 2013, 62, 499-513. [CrossRef]

57. Wang, R.; Dorp, L.V.; Shaw, L.P.; Bradley, P.; Wang, Q.; Wang, X.; Jin, L.; Zhang, Q.; Liu, Y.; Rieux, A.; et al. The global distribution and spread of the mobilized colistin resistance gene mcr-1. Nat. Commun. 2018, 9, 1179. [CrossRef] 